\title{
GOAL-ORIENTED ADAPTIVITY IN CONTROL CONSTRAINED OPTIMAL CONTROL OF PARTIAL DIFFERENTIAL EQUATIONS
}

\author{
M. HINTERMÜLLER AND R.H.W. HOPPE
}

\begin{abstract}
Dual-weighted goal-oriented error estimates for a class of pointwise control constrained optimal control problems for second order elliptic partial differential equations are derived. It is demonstrated that the constraints give rise to a primal-dual weighted error term representing the mismatch in the complementarity system due to discretization. Also, for the new error estimate a posteriori error estimators for the $L^{2}$-norm of the solution and its adjoint are derived.
\end{abstract}

\section{INTRODUCTION}

In many computations involving the discretization of (partial) differential equations or variational inequalities one is interested in the accurate evaluation of some target quantity. This might be the value of the solution of a partial differential equation (PDE) at some reference point in the domain of interest, a physically relevant quantity such as the drag in airfoil design, or, in optimal control, the value of the objective function at the solution of the underlying minimization problem. Highly accurate numerical evaluations of these targets can be guaranteed by using uniform meshes with a small mesh size $h$. This, however, usually represents a significant computational challenge due to the resulting large scale of the discrete problems. Therefore, one seeks to adaptively refine the meshes with the goal of achieving a desired accuracy in the evaluation of the output quantity of interest while keeping the computational cost as small as possible.

For this purpose, recently for (systems of) partial differential equations an approach based on dual weighted residual-based error estimates was proposed. Here we point to the pioneering work summarized in $[1,3]$ and the references therein; see also [4] for related literature. It essentially relies on employing the dual problem of the underlying system with the target on the right hand side. In fact, let $A$ denote some possibly nonlinear partial

1991 Mathematics Subject Classification. 49K20, 65K10, 65N30, 65N50.

Key words and phrases. adaptive finite element method, a posteriori error estimate, control constraints, goal-oriented adaptivity, PDE-constrained optimization.

The first author (M.H.) gratefully acknowledges financial support from the Austrian Science Fund FWF under START-program Y305 "Interfaces and Free Boundaries". The second author (R.H.W.H.) has been partially supported by the NSF under grant no. DMS-0411403 and grant no. DMS-0511611. 
differential operator and let $f$ be some fixed data. Then, in some abstract form, the primal problem (or PDE) is given by

$$
A(y)=f \text {. }
$$

Let $y_{h}$ be the result of a Galerkin finite element discretization of the underlying problem. If $G(\cdot)$ represents some desired target quantity (or goal), then the dual approach consists in considering

$$
A^{\prime}\left(y_{h}\right)^{\star} p_{h}=G(\cdot)
$$

from which an a posteriori error estimate of the type

$$
\left|G(y)-G\left(y_{h}\right)\right| \leq \sum_{T \in \mathbb{T}_{h}} \mathfrak{p}_{T}\left(y_{h}\right) \mathfrak{d}_{T}\left(p_{h}\right)
$$

is derived. Above, $A^{\prime}(\cdot)^{\star}$ is the dual operator of the Frechét-derivative $A^{\prime}(\cdot)$ of $A(\cdot)$. Further, $\mathbb{T}_{h}=\{T\}$ denotes a computational mesh consisting of elements $T$, and $\mathfrak{p}_{T}$ and $\mathfrak{d}_{T}$ stand for the primal residual and the dual weight on each cell $T$, respectively.

In [2] this concept was transferred to PDE-constrained optimal control problems of the type

$$
\text { minimize } J(y, u) \quad \text { subject to } \quad A(y)=f+B(u)
$$

where $(y, u)$ denotes the state-control pair and $B$ models the control impact. The first order optimality system of $\left(\mathrm{P}_{0}\right)$ can be formally written as

$$
\begin{aligned}
A(y)-B(u) & =f, \\
J_{y}(y, u)+A^{\prime}(y)^{\star} p & =0, \\
J_{u}(y, u)-B^{\prime}(u)^{\star} p & =0 .
\end{aligned}
$$

Here, $J_{y}$ and $J_{u}$ are the partial derivatives of $J$ with respect to $y$ and $u$, respectively. The variable $p$ is called the adjoint state. Often, (3c) results in an algebraic equation, while (3a)-(3b) forms a primal-dual pair of PDEs similar to (1)-(2). Since (3a)-(3b) represents a system of PDEs the dual weighted approach can be readily carried over to this optimal control setting.

The situation, however, changes significantly if, in addition to the PDE constraint in $\left(\mathrm{P}_{0}\right)$, one has to account for pointwise almost everywhere constraints on the control variable. In this case, the resulting problem becomes

$$
\left(\mathrm{P}_{c}\right) \begin{cases}\text { minimize } & J(y, u) \\ \text { subject to } & A(y)=f+B(u), \\ & a \leq u \leq b \quad \text { almost everywhere (a.e.) on } \Omega_{C} \subset \Omega,\end{cases}
$$

where $\Omega \subset \mathbb{R}^{n}$ denotes some suitable domain with $\Omega_{C} \neq \emptyset$ a measurable subset, and where $a<b$ are given bounds. The corresponding first order necessary optimality system now involves a variational inequality:

$$
\begin{aligned}
A(y)-B(u) & =f \\
J_{y}(y, u)+A^{\prime}(y)^{\star} p & =0 \\
\left\langle J_{u}(y, u)-B^{\prime}(u)^{\star} p, v-u\right\rangle & \geq 0 \quad \forall v \in U^{\mathrm{ad}}, u \in U^{\mathrm{ad}}
\end{aligned}
$$


where the set

$$
U^{\mathrm{ad}}=\{v: a \leq v \leq b\}
$$

represents the feasible controls, and $\langle\cdot, \cdot\rangle$ denotes a suitable duality pairing. The variational inequality induces some nonsmoothness in the first order optimality system. This can be seen best when defining the Lagrange multiplier $\lambda$ pertinent to the pointwise constraints via

$$
J_{u}(y, u)-B^{\prime}(u)^{\star} p+\lambda=0
$$

and, assuming that $\lambda$ permits a pointwise interpretation,

$$
\lambda \geq 0 \quad \text { a.e. on }\{u=b\}, \quad \lambda \leq 0 \quad \text { a.e. on }\{u=a\}, \quad \lambda=0 \quad \text { else. }
$$

The conditions in (6) represent the so-called complementarity system. It can be written equivalently as

$$
\lambda=\min \{0, \lambda+\sigma(u-a)\}+\max \{0, \lambda+\sigma(u-b)\},
$$

where $\sigma>0$ is an arbitrarily fixed real and the max- and min-operations are understood in the pointwise sense. From (7) the nonsmoothness involved in the first order necessary optimality conditions becomes apparent. Of course, suitable a posteriori error concepts have to reflect this situation in order to accurately resolve the influence of the constraints on the solution of the optimal control problem.

In this paper, our starting point will be a sufficiently general model problem class of the type $\left(\mathrm{P}_{c}\right)$. Based on the Lagrange function

$$
\mathcal{L}(y, u, p, \lambda)=J(y, u)+\langle A(y)-f-B(u), p\rangle+(u-b, \lambda)
$$

of $\left(\mathrm{P}_{c}\right)$, for convenience written here for a unilaterally constrained version of the minimization problem, and with the objective function as the goal, we derive an error representation of the type

$$
\begin{aligned}
J(y, u)-J\left(y_{h}, u_{h}\right)=-\frac{1}{2}\langle & \left.\nabla_{x x} \mathcal{L}\left(x_{h}, \lambda_{h}\right)\left(x_{h}-x\right), x_{h}-x\right\rangle+\left(u_{h}-b, \lambda\right) \\
& +\operatorname{osc}_{h}+r\left(x_{h}, x\right)
\end{aligned}
$$

with $x=(p, y, u)$ and its discretized version $x_{h}=\left(p_{h}, y_{h}, u_{h}\right)$, respectively, and $(\cdot, \cdot)$ some inner product. Further, $\mathrm{osc}_{h}$ represents data oscillations and $r$ is the remainder term resulting from a Taylor expansion of $\mathcal{L}$. In a second step we then estimate the term due to the inequality constraints and utilize the a posteriori error estimators derived in [5] in order to obtain a computable error representation.

The rest of the paper is organized as follows: In the next section we derive our new dual-weighted residual-based error estimator for a representative control constrained optimal control model problem. Section 3 is devoted to possible extensions. In fact, we study the bilaterally constrained case, a class of nonlinear governing equations, and alternative concepts for obtaining a posteriori estimates pertinent to the complementarity system. In the appendix, for our constrained optimal control problem we derive a new a posteriori error estimate with respect to the $L^{2}$-norm. 
Notation. Throughout we use $\|\cdot\|_{0, \Omega}$ and $(\cdot, \cdot)_{0, \Omega}$ for the usual $L^{2}(\Omega)$ norm and $L^{2}(\Omega)$-inner product, respectively. For convenience, with respect to the notation we shall not distinguish between the norm, respectively inner product, for scalar-valued or vector-valued arguments. We also use $(\cdot, \cdot)_{0, \mathcal{S}}$, which is the $L^{2}(\mathcal{S})$-inner product over a (measurable) subset $\mathcal{S} \subset \Omega$. By | $\left.\cdot\right|_{1, \Omega}$ we denote the $H^{1}(\Omega)$-seminorm $|y|_{1, \Omega}=\|\nabla y\|_{0, \Omega}$, which, by the Poincaré-Friedrichs-inequality, is a norm on $H_{0}^{1}(\Omega)$. The norm in $H^{1}(\Omega)$ is written as $\|\cdot\|_{1, \Omega}$. By $\mathbb{T}_{h}=\mathbb{T}_{h}(\Omega)$ we denote a shape regular finite element triangulation of the domain $\Omega$. The subscript $h=\max \left\{\operatorname{diam}(T) \mid T \in \mathbb{T}_{h}\right\}$ indicates the mesh size of $\mathbb{T}_{h}$.

\section{Residual Based ERror estimate}

For deriving the structure of the new error estimate due to the inequality constraints, we consider the model problem

$$
\left\{\begin{array}{l}
\operatorname{minimize} J(y, u):=\frac{1}{2}\|y-z\|_{0, \Omega}^{2}+\frac{\alpha}{2}\|u\|_{0, \Omega}^{2} \\
\text { over } \quad(y, u) \in H_{0}^{1}(\Omega) \times L^{2}(\Omega) \\
\text { subject to }-\Delta y=u+f \\
\quad u \leq b \quad \text { a.e. in } \Omega,
\end{array}\right.
$$

which is a particular instance of $\left(\mathrm{P}_{c}\right)$. The domain $\Omega \in \mathbb{R}^{2}$ is assumed to be bounded and polygonal with boundary $\Gamma:=\partial \Omega$. For the data we suppose $z, b, f \in L^{2}(\Omega)$ and $\alpha>0$. It is well-known that $(\mathrm{P})$ admits a unique solution $\left(y^{*}, u^{*}\right) \in H_{0}^{1}(\Omega) \times L^{2}(\Omega)$. Moreover, the optimal solution is characterized by the existence of an adjoint state $p^{*} \in H_{0}^{1}(\Omega)$ and a Lagrange multiplier $\lambda^{*} \in L^{2}(\Omega)$ which satisfy the first order necessary (and in this case also sufficient) conditions

$$
\begin{aligned}
& -\Delta y^{*}=u^{*}+f \\
& -\Delta p^{*}+y^{*}=z \\
& \alpha u^{*}+\lambda^{*}-p^{*}=0, \\
& u^{*} \leq b, \lambda^{*} \geq 0,\left(u^{*}-b, \lambda^{*}\right)_{0, \Omega}=0 .
\end{aligned}
$$

We define the Lagrange functional $\mathcal{L}: H_{0}^{1}(\Omega) \times L^{2}(\Omega) \times H_{0}^{1}(\Omega) \times L^{2}(\Omega) \rightarrow$ $\mathbb{R}$ pertinent to $(\mathrm{P})$ as

$$
\mathcal{L}(y, u, p, \lambda)=J(y, u)+(\nabla y, \nabla p)_{0, \Omega}-(u+f, p)_{0, \Omega}+(u-b, \lambda)_{0, \Omega} .
$$

For convenience we use $x:=(p, y, u), x^{*}=\left(p^{*}, y^{*}, u^{*}\right)$ and $X=P \times Y \times L=$ $H_{0}^{1}(\Omega) \times H_{0}^{1}(\Omega) \times L^{2}(\Omega)$. Obviously, the weak form of $(8 \mathrm{a})-(8 \mathrm{~b})$ and $(8 \mathrm{c})$ of the optimality system (8) is equivalent to

$$
\nabla_{x} \mathcal{L}\left(x^{*}, \lambda^{*}\right)(\varphi)=0 \quad \forall \varphi \in X
$$

Let $X_{h} \subset X$, with $X_{h}=P_{h} \times Y_{h} \times L_{h}$, denote a finite dimensional subspace with the subscript $h$ indicating the mesh size of discretization obtained 
by a standard Galerkin method, and let $\lambda_{h} \in L_{h} \subset L^{2}(\Omega)$ denote the discrete (finite dimensional) counterpart of $\lambda$ (analogously for $\lambda^{*}$ ). The finite dimensional version of $(8)$ reads

$$
\begin{aligned}
& \nabla_{x} \mathcal{L}_{h}\left(x_{h}^{*}, \lambda_{h}^{*}\right)\left(\varphi_{h}\right)=0 \quad \forall \varphi_{h} \in X_{h}, \\
& u_{h}^{*} \leq b_{h}, \quad \lambda_{h}^{*} \geq 0, \quad\left(u_{h}^{*}-b_{h}, \lambda_{h}^{*}\right)_{0, \Omega}=0,
\end{aligned}
$$

where the discrete Lagrange function is given by

$$
\begin{aligned}
\mathcal{L}_{h}\left(x_{h}, \lambda_{h}\right)= & J_{h}\left(y_{h}, u_{h}\right)+\left(\nabla y_{h}, \nabla p_{h}\right)_{0, \Omega}-\left(u_{h}+f_{h}, p_{h}\right)_{0, \Omega} \\
& +\left(u_{h}-b_{h}, \lambda_{h}\right)_{0, \Omega}
\end{aligned}
$$

with $J_{h}\left(y_{h}, u_{h}\right)=\frac{1}{2}\left\|y_{h}-z_{h}\right\|_{0, \Omega}^{2}+\frac{\alpha}{2}\left\|u_{h}\right\|_{0, \Omega}^{2}$. Observe that the pointwise representation $(8 \mathrm{c})$ in the discrete setting reads

$$
\alpha u_{h}^{*}+\lambda_{h}^{*}-M_{h} p_{h}^{*}=0,
$$

where $M_{h}$ represents a projection operator from $P_{h}$ onto $L_{h}$.

Further note that for $x \in X, \lambda \in L^{2}(\Omega)$ and $x_{h} \in X_{h}, \lambda_{h} \in L_{h}$

$$
\begin{aligned}
\mathcal{L}\left(x, \lambda_{h}\right) & =\mathcal{L}(x, \lambda)+\left(u-b, \lambda_{h}-\lambda\right)_{0, \Omega}, \\
\nabla_{x} \mathcal{L}\left(x_{h}, \lambda_{h}\right)\left(\varphi_{h}\right) & =\nabla_{x} \mathcal{L}\left(x_{h}, \lambda\right)\left(\varphi_{h}\right)+\left(\delta u_{h}, \lambda_{h}-\lambda\right)_{0, \Omega}
\end{aligned}
$$

for all $\left(\delta p_{h}, \delta y_{h}, \delta u_{h}\right)=\varphi_{h} \in X_{h}$. Moreover, for our model problem (P) the second derivative of $\mathcal{L}$ with respect to $x$ does not depend on $x$ and $\lambda$. Thus, we can write $\nabla_{x x} \mathcal{L}(\varphi, \hat{\varphi})$ instead of $\nabla_{x x} \mathcal{L}(x, \lambda)(\varphi, \hat{\varphi})$. Similar observations hold true for $\mathcal{L}_{h}$. Due to $X_{h} \subset X$, we have for $\varphi_{h}=\left(\delta p_{h}, \delta y_{h}, \delta u_{h}\right) \in X_{h}$

$$
\begin{aligned}
0= & \nabla_{x} \mathcal{L}\left(x^{*}, \lambda^{*}\right)\left(\varphi_{h}\right) \\
= & \nabla_{x} \mathcal{L}\left(x_{h}^{*}, \lambda^{*}\right)\left(\varphi_{h}\right)+\nabla_{x x} \mathcal{L}\left(x^{*}-x_{h}^{*}, \varphi_{h}\right) \\
= & \nabla_{x} \mathcal{L}\left(x_{h}^{*}, \lambda_{h}^{*}\right)\left(\varphi_{h}\right)+\left(\delta u_{h}, \lambda^{*}-\lambda_{h}^{*}\right)_{0, \Omega}+\nabla_{x x} \mathcal{L}\left(x^{*}-x_{h}^{*}, \varphi_{h}\right) \\
= & \nabla_{x} \mathcal{L}_{h}\left(x_{h}^{*}, \lambda_{h}^{*}\right)\left(\varphi_{h}\right)-\left(f-f_{h}, \delta p_{h}\right)_{0, \Omega}-\left(z-z_{h}, \delta y_{h}\right)_{0, \Omega} \\
& +\left(\delta u_{h}, \lambda^{*}-\lambda_{h}^{*}\right)_{0, \Omega}+\nabla_{x x} \mathcal{L}\left(x^{*}-x_{h}^{*}, \varphi_{h}\right) \\
= & \left(\delta u_{h}, \lambda^{*}-\lambda_{h}^{*}\right)_{0, \Omega}+\nabla_{x x} \mathcal{L}\left(x^{*}-x_{h}^{*}, \varphi_{h}\right)-\left(f-f_{h}, \delta p_{h}\right)_{0, \Omega} \\
& -\left(z-z_{h}, \delta y_{h}\right)_{0, \Omega} .
\end{aligned}
$$

From this we further derive the relations

$$
\begin{aligned}
& \nabla_{x x} \mathcal{L}\left(x_{h}^{*}-x^{*}, x_{h}^{*}-x^{*}\right)= \\
& \quad=\nabla_{x x} \mathcal{L}\left(x_{h}^{*}-x^{*}, x_{h}^{*}-x^{*}+\varphi_{h}\right)-\left(\delta u_{h}, \lambda^{*}-\lambda_{h}^{*}\right)_{0, \Omega} \\
& \quad+\left(f-f_{h}, \delta p_{h}\right)_{0, \Omega}+\left(z-z_{h}, \delta y_{h}\right)_{0, \Omega} \\
& \quad \nabla_{x} \mathcal{L}\left(x_{h}^{*}, \lambda^{*}\right)\left(x^{*}-x_{h}^{*}-\varphi_{h}\right)=\nabla_{x x} \mathcal{L}\left(x_{h}^{*}-x^{*}, x^{*}-x_{h}^{*}-\varphi_{h}\right)
\end{aligned}
$$

and also

$$
\begin{aligned}
& \nabla_{x} \mathcal{L}\left(x_{h}^{*}, \lambda_{h}^{*}\right)\left(x^{*}-x_{h}^{*}-\varphi_{h}\right)= \\
& \quad=\nabla_{x} \mathcal{L}\left(x^{*}, \lambda_{h}^{*}\right)\left(x^{*}-x_{h}^{*}-\varphi_{h}\right)+\nabla_{x x} \mathcal{L}\left(x_{h}^{*}-x^{*}, x^{*}-x_{h}^{*}-\varphi_{h}\right) \\
& \quad=\left(\lambda_{h}^{*}-\lambda^{*}, u^{*}-u_{h}^{*}-\delta u_{h}\right)_{0, \Omega}+\nabla_{x x} \mathcal{L}\left(x_{h}^{*}-x^{*}, x^{*}-x_{h}^{*}-\varphi_{h}\right) .
\end{aligned}
$$

These preliminary results are now used to prove the following theorem. 
Theorem 2.1. Let $\left(x^{*}, \lambda^{*}\right) \in X \times L^{2}(\Omega)$ and $\left(x_{h}^{*}, \lambda_{h}^{*}\right) \in X_{h} \times L_{h}$ denote the solution of (8) and its finite dimensional counterpart (11). Then

$$
\begin{aligned}
J\left(y^{*}, u^{*}\right)-J_{h}\left(y_{h}^{*}, u_{h}^{*}\right)=- & \frac{1}{2} \nabla_{x x} \mathcal{L}\left(x_{h}^{*}-x^{*}, x_{h}^{*}-x^{*}\right) \\
& +\left(u_{h}^{*}-b, \lambda^{*}\right)_{0, \Omega}+\operatorname{osc}_{h}\left(x_{h}^{*}\right),
\end{aligned}
$$

where the oscillations $\operatorname{osc}_{h}\left(x_{h}^{*}\right)$ are given by

$$
\operatorname{osc}_{h}\left(x_{h}^{*}\right)=\left(y_{h}^{*}-z_{h}, z_{h}-z\right)_{0, \Omega}+\frac{1}{2}\left\|z-z_{h}\right\|_{0, \Omega}^{2}+\left(f_{h}-f, p_{h}^{*}\right)_{0, \Omega} .
$$

Proof. Observe that $J\left(y^{*}, u^{*}\right)=\mathcal{L}\left(x^{*}, \lambda^{*}\right)$ and $J_{h}\left(y_{h}^{*}, u_{h}^{*}\right)=\mathcal{L}_{h}\left(x_{h}^{*}, \lambda_{h}^{*}\right)$. Using Taylor expansions and (14)-(15) we obtain

$$
\begin{aligned}
J\left(y^{*}, u^{*}\right)- & J_{h}\left(y_{h}^{*}, u_{h}^{*}\right)=\mathcal{L}\left(x^{*}, \lambda^{*}\right)-\mathcal{L}_{h}\left(x_{h}^{*}, \lambda_{h}^{*}\right)= \\
= & \mathcal{L}\left(x^{*}, \lambda^{*}\right)-\mathcal{L}_{h}\left(x^{*}, \lambda_{h}^{*}\right)-\nabla_{x} \mathcal{L}_{h}\left(x^{*}, \lambda_{h}^{*}\right)\left(x_{h}^{*}-x^{*}\right) \\
& -\frac{1}{2} \nabla_{x x} \mathcal{L}_{h}\left(x_{h}^{*}-x^{*}, x_{h}^{*}-x^{*}\right) \\
= & J\left(y^{*}, u^{*}\right)-J_{h}\left(y^{*}, u^{*}\right)+\left(f_{h}-f, p^{*}\right)_{0, \Omega}-\left(u^{*}-b_{h}, \lambda_{h}^{*}\right)_{0, \Omega} \\
& -\nabla_{x} \mathcal{L}_{h}\left(x^{*}, \lambda_{h}^{*}\right)\left(x_{h}^{*}-x^{*}\right)-\frac{1}{2} \nabla_{x x} \mathcal{L}_{h}\left(x_{h}^{*}-x^{*}, x_{h}^{*}-x^{*}\right) \\
= & \operatorname{osc}_{h}\left(x_{h}^{*}\right)-\left(u^{*}-b_{h}, \lambda_{h}^{*}\right)_{0, \Omega}-\nabla_{x} \mathcal{L}\left(x^{*}, \lambda_{h}^{*}\right)\left(x_{h}^{*}-x^{*}\right) \\
& -\frac{1}{2} \nabla_{x x} \mathcal{L}_{h}\left(x_{h}^{*}-x^{*}, x_{h}^{*}-x^{*}\right) \\
= & \operatorname{osc}_{h}\left(x_{h}^{*}\right)-\left(u^{*}-u_{h}^{*}, \lambda_{h}^{*}\right)_{0, \Omega}+\left(\lambda^{*}-\lambda_{h}^{*}, u_{h}^{*}-u^{*}\right)_{0, \Omega} \\
& -\frac{1}{2} \nabla_{x x} \mathcal{L}_{h}\left(x_{h}^{*}-x^{*}, x_{h}^{*}-x^{*}\right) \\
= & \operatorname{osc}_{h}\left(x_{h}^{*}\right)+\left(\lambda^{*}, u_{h}^{*}-b\right)_{0, \Omega}-\frac{1}{2} \nabla_{x x} \mathcal{L}_{h}\left(x_{h}^{*}-x^{*}, x_{h}^{*}-x^{*}\right),
\end{aligned}
$$

where we also used the complementarity relations (8d) and (11b) as well as (10) and (11a).

Assume, for the moment, that $\lambda^{*}=0$ and $\lambda_{h}^{*}=0$, i.e., the continuous and the discrete control constraints are inactive, respectively. Then we infer from (17)

$$
\begin{aligned}
\nabla_{x x} \mathcal{L}\left(x_{h}^{*}-x^{*}, x_{h}^{*}-x^{*}\right)=\nabla_{x x} \mathcal{L}\left(x_{h}^{*}-x^{*}, x_{h}^{*}-x^{*}+\varphi_{h}\right) \\
+\left(f-f_{h}, \delta p_{h}\right)_{0, \Omega}+\left(z-z_{h}, \delta y_{h}\right)_{0, \Omega}
\end{aligned}
$$

and further

$$
\begin{aligned}
J\left(y^{*}, u^{*}\right)-J_{h} & \left(y_{h}^{*}, u_{h}^{*}\right)=\frac{1}{2} \nabla_{x} \mathcal{L}_{h}\left(x_{h}, \lambda_{h}\right)\left(x^{*}-x_{h}^{*}-\varphi_{h}\right) \\
& +\frac{1}{2}\left(f_{h}-f, p^{*}-p_{h}^{*}\right)_{0, \Omega}+\frac{1}{2}\left(z_{h}-z, y^{*}-y_{h}^{*}\right)_{0, \Omega} \\
& +\operatorname{osc}_{h}\left(x_{h}^{*}\right)
\end{aligned}
$$

due to (19). This corresponds to the result in [2, Proposition 4.1] for the unconstrained version of $(\mathrm{P})$. 
If $b_{h} \leq b$ a.e. in $\Omega$, then (20) implies

$$
J\left(y^{*}, u^{*}\right) \leq J_{h}\left(y_{h}^{*}, u_{h}^{*}\right)+\operatorname{osc}_{h}\left(x^{*}, x_{h}^{*}\right) .
$$

Next we interpret the new, second term in the right hand side of (20). For this purpose we define the active set $\mathcal{A}^{*}$ and the inactive set $\mathcal{I}^{*}$ at the optimal solution $\left(x^{*}, \lambda^{*}\right)$ of $(\mathrm{P})$ by

$$
\mathcal{A}^{*}:=\left\{x \in \Omega: u^{*}(x)=b(x)\right\}, \quad \mathcal{I}^{*}:=\Omega \backslash \mathcal{A}^{*} .
$$

Analogously we define the discrete counterparts $\mathcal{A}_{h}^{*}$ and $\mathcal{I}_{h}^{*}$, respectively. Obviously, $u^{*}<b$ a.e. in $\mathcal{I}^{*}$. By $(8 \mathrm{~d})$, this implies $\lambda^{*}=0$ a.e. in $\mathcal{I}^{*}$. Therefore, the term $\left(u_{h}^{*}-b, \lambda^{*}\right)_{0, \Omega}$ satisfies

$$
\left(u_{h}^{*}-b, \lambda^{*}\right)_{0, \Omega}=\left(u_{h}^{*}-b_{h}, \lambda^{*}\right)_{0, \mathcal{A}^{*} \cap \mathcal{I}_{h}^{*}}+\left(b_{h}-b, \lambda^{*}\right)_{0, \mathcal{A}^{*}}
$$

The right hand side above reflects the error in complementarity. In fact, the second term represents the data oscillation in the bound in the active set weighted by the continuous Lagrange multiplier. For this term we introduce the notation

$$
\operatorname{osc}_{h}^{\mathcal{A}^{*}}\left(b ; \lambda^{*}\right):=\left(b_{h}-b, \lambda^{*}\right)_{0, \mathcal{A}^{*}}
$$

The first term captures a primal-dual weighted mismatch in complementarity in $\mathcal{A}^{*} \cap \mathcal{I}_{h}^{*}$.

Let $i_{h}:=\left(i_{h}^{p}, i_{h}^{y}, i_{h}^{u}\right)$ be an interpolation operator such that $i_{h} x \in X_{h}$ for $x \in X$. Moreover, for $y, p \in H_{0}^{1}(\Omega)$ there exist $i_{h}^{p}$ and $i_{h}^{y}$ such that $\max \left\{\left\|i_{h}^{p} p-p\right\|_{H^{1}},\left\|i_{h}^{y} y-y\right\|_{H^{1}}\right\} \rightarrow 0$ for $h \rightarrow 0$. In connection with Theorem 2.1 we have the following result.

Theorem 2.2. Let the assumptions of Theorem 2.1 be satisfied. Then

$$
\begin{aligned}
J\left(y^{*}, u^{*}\right)- & J_{h}\left(y_{h}^{*}, u_{h}^{*}\right)= \\
= & -\frac{1}{2}\left(\left(y_{h}^{*}-z_{h}, i_{h}^{y} y^{*}-y^{*}\right)_{0, \Omega}+\left(\nabla\left(i_{h}^{y} y^{*}-y^{*}\right), \nabla p_{h}^{*}\right)_{0, \Omega}\right. \\
& +\left(\nabla\left(i_{h}^{p} p^{*}-p^{*}\right), \nabla y_{h}^{*}\right)_{0, \Omega}-\left(u_{h}^{*}+f_{h}, i_{h}^{p} p^{*}-p^{*}\right)_{0, \Omega} \\
& \left.+\left(M_{h} p_{h}^{*}-p_{h}^{*}, i_{h}^{u} u^{*}-u^{*}\right)_{0, \Omega}\right) \\
& +\frac{1}{2}\left[\left(b-u_{h}^{*}, \lambda^{*}\right)_{0, \Omega}+\left(b_{h}-u^{*}, \lambda_{h}^{*}\right)_{0, \Omega}\right]+\frac{1}{2}\left(f-f_{h}, p_{h}^{*}-p^{*}\right)_{0, \Omega} \\
& +\frac{1}{2}\left(z-z_{h}, y_{h}^{*}-y^{*}\right)_{0, \Omega}+\operatorname{osc}_{h}\left(x_{h}^{*}\right) .
\end{aligned}
$$


Proof. Utilizing (17)-(18) and considering $\varphi_{h}=\left(\delta p_{h}, \delta y_{h}, \delta u_{h}\right) \in X_{h}$ we obtain

$$
\begin{aligned}
J\left(y^{*}, u^{*}\right)- & J_{h}\left(y_{h}^{*}, u_{h}^{*}\right)=\frac{1}{2} \nabla_{x x} \mathcal{L}\left(x, \lambda_{h}^{*}\right)\left(x^{*}-x_{h}^{*}, x_{h}^{*}-x^{*}+\varphi_{h}\right) \\
& +\frac{1}{2}\left(\delta u_{h}, \lambda^{*}-\lambda_{h}^{*}\right)_{0, \Omega}+\frac{1}{2}\left(f_{h}-f, \delta p_{h}\right)_{0, \Omega}+\frac{1}{2}\left(z_{h}-z, \delta y_{h}\right)_{0, \Omega} \\
& +\left(u_{h}^{*}-b, \lambda^{*}\right)_{0, \Omega}+\operatorname{osc}_{h}\left(x_{h}^{*}\right) \\
= & -\frac{1}{2} \nabla_{x} \mathcal{L}\left(x_{h}^{*}, \lambda_{h}^{*}\right)\left(x_{h}^{*}-x^{*}+\varphi_{h}\right)+\frac{1}{2}\left(\lambda_{h}^{*}-\lambda^{*}, u_{h}^{*}-u^{*}\right)_{0, \Omega} \\
& +\frac{1}{2}\left(f_{h}-f, \delta p_{h}\right)_{0, \Omega}+\frac{1}{2}\left(z_{h}-z, \delta y_{h}\right)_{0, \Omega}+\operatorname{osc}_{h}\left(x_{h}^{*}\right) \\
= & -\frac{1}{2} \nabla_{x} \mathcal{L}_{h}\left(x_{h}^{*}, \lambda_{h}^{*}\right)\left(x_{h}^{*}-x^{*}+\varphi_{h}\right)+\frac{1}{2}\left(\lambda_{h}^{*}-\lambda^{*}, u_{h}^{*}-u^{*}\right)_{0, \Omega} \\
& +\frac{1}{2}\left(f-f_{h}, p_{h}^{*}-p^{*}\right)_{0, \Omega}+\frac{1}{2}\left(z-z_{h}, y_{h}^{*}-y^{*}\right)_{0, \Omega}+\operatorname{osc}_{h}\left(x_{h}^{*}\right) .
\end{aligned}
$$

Choosing $\varphi_{h}=\left(i_{h}^{p} p^{*}-p_{h}^{*}, i_{h}^{y} y^{*}-y_{h}^{*}, i_{h}^{u} u^{*}-u_{h}^{*}\right) \in X_{h}$ and using complementary slackness, we continue

$$
\begin{aligned}
J\left(y^{*}, u^{*}\right) & -J_{h}\left(y_{h}^{*}, u_{h}^{*}\right)=-\frac{1}{2} \nabla_{x} \mathcal{L}_{h}\left(x_{h}^{*}, \lambda_{h}^{*}\right)\left(i_{h} x^{*}-x^{*}\right)+\frac{1}{2}\left[\left(\lambda_{h}^{*}, b_{h}-u^{*}\right)_{0, \Omega}\right. \\
& \left.+\left(\lambda^{*}, b-u_{h}^{*}\right)_{0, \Omega}\right]+\frac{1}{2}\left(f-f_{h}, p_{h}^{*}-p^{*}\right)_{0, \Omega}+\frac{1}{2}\left(z-z_{h}, y_{h}^{*}-y^{*}\right)_{0, \Omega} \\
& +\operatorname{osc}_{h}\left(x_{h}^{*}\right) .
\end{aligned}
$$

The assertion now follows from (9) and $u_{h}^{*}-M_{h} p_{h}^{*}+\lambda_{h}^{*}=0$ a.e. in $\Omega$.

This result is interesting in several ways:

(i) For $\left\|M_{h} p_{h}-p_{h}\right\|_{0, \Omega} \rightarrow 0$ as $h \rightarrow 0$ sufficiently fast, only the convergence properties implied by $i_{h}^{p}$ and $i_{h}^{y}$ are required for obtaining an a posteriori error estimate based on (23). Since $y^{*}$ and $p^{*}$ solve elliptic partial differential equations they usually enjoy more regularity than $u^{*}$ and $\lambda^{*}$.

(ii) The term in brackets on the right hand side in (23) is again related to errors coming from complementary slackness. The first term of the sum can be interpreted as before, while the second term of the sum reflects the symmetric case, i.e.,

$$
\left(b_{h}-u^{*}, \lambda_{h}^{*}\right)_{0, \Omega}=\left(b-u^{*}, \lambda_{h}^{*}\right)_{0, \mathcal{A}_{h}^{*} \cap \mathcal{I}^{*}}+\left(b_{h}-b, \lambda_{h}^{*}\right)_{0, \mathcal{A}_{h}^{*}},
$$

Hence, the first term of the right hand side above represents the primal-dual weighted mismatch in complementarity in $\mathcal{I}^{*} \cap \mathcal{A}_{h}^{*}$, while the second term denotes the data oscillation on $\mathcal{A}_{h}^{*}$ weighted by the discrete multiplier, i.e.,

$$
\operatorname{osc}_{h}^{\mathcal{A}_{h}^{*}}\left(b ; \lambda_{h}^{*}\right):=\left(b_{h}-b, \lambda_{h}^{*}\right)_{0, \mathcal{A}_{h}^{*}} .
$$

Of course, (23) is not immediately amenable to numerical realization since $u^{*}$ and $\lambda^{*}$ are involved. Before we tackle this point, let us first state a 
posteriori error bounds for the control and the adjoint state which were derived in [5]. A coarser estimate was established in [7]. Recall that $U^{a d}$ denotes the set of admissible controls, and let $U_{h}^{a d}$ be its discretization. Then the following a posteriori error estimates hold true:

(24a) $\max \left(\left\|\lambda^{*}-\lambda_{h}^{*}\right\|_{0, \Omega}^{2},\left\|u^{*}-u_{h}^{*}\right\|_{0, \Omega}^{2}\right) \leq C_{1}^{2} \eta_{1}^{2}+C_{2}^{2} \eta_{2}^{2}+C_{b}^{2} \mu_{h}^{2}(b)$,

$$
\left|p^{*}-p_{h}^{*}\right|_{1, \Omega}^{2} \leq C_{2}^{2} \eta_{2}^{2}+C_{z}^{2} \operatorname{osc}_{h}^{2}(z)
$$

In what follows we also use

$$
C_{3}^{2} \eta_{3}^{2}:=C_{1}^{2} \eta_{1}^{2}+C_{2}^{2} \eta_{2}^{2}+C_{b}^{2} \mu_{h}^{2}(b) \quad \text { and } \quad C_{4}^{2} \eta_{4}^{2}:=C_{2}^{2} \eta_{2}^{2}+C_{z}^{2} \operatorname{osc}_{h}^{2}(z) \text {. }
$$

Here and below, $C_{i}>0, i=1,2,3,4$, denote constants which depend on $\alpha$, $\Omega$ and the shape regularity of $\mathbb{T}_{h}$. The error bounds $\eta_{1}$ and $\eta_{2}$ are defined as

$$
\begin{aligned}
& \eta_{1}^{2}=\sum_{T} \int_{T} h_{T}^{2}\left(p_{h}^{*}-M_{h} p_{h}^{*}\right)^{2} \\
& \eta_{2}^{2}=\sum_{T} \int_{T} h_{T}^{2}\left(f+u_{h}^{*}+\Delta y_{h}^{*}\right)^{2}+\sum_{F} \int_{F} h_{F}\left[\nabla y_{h}^{*} \cdot n\right]^{2} \\
& \quad+\sum_{T} \int_{T} h_{T}^{2}\left(z-y_{h}^{*}+\Delta p_{h}^{*}\right)^{2}+\sum_{F} \int_{F} h_{F}\left[\nabla p_{h}^{*} \cdot n\right]^{2} .
\end{aligned}
$$

Further the data oscillations

$$
\begin{aligned}
\mu_{h}^{2}(b) & =\sum_{T \in \mathbb{T}_{h}}\left\|b-b_{h}\right\|_{0, T}^{2}, \\
\operatorname{osc}_{h}^{2}(z) & =\sum_{T \in \mathbb{T}_{h}} h_{T}^{2}\left\|z-z_{h}\right\|_{0, T}^{2}
\end{aligned}
$$

are involved.

Above, $T$ denotes an element of the triangulation $\mathbb{T}_{h}$ of $\Omega$. Further, $F$ denotes a face of $T$, and $h_{F}$ is the maximal diameter of the face $F$. Moreover, $\left[\nabla y_{h}^{*} \cdot n\right]$ is the normal derivative jump over an interior face $F$. As noted before, the operator $M_{h}$ represents the projection of a mesh function in $P_{h}$ (= $Y_{h}$, typically in our context) onto $L_{h}$. If $L_{h}$ is given by

$$
L_{h}=\left\{u_{h} \in L^{2}(\Omega):\left.u_{h}\right|_{T} \in P_{0}(T), T \in \mathbb{T}_{h}\right\},
$$

i.e., the function $u_{h}$ is piecewise constant on $\mathbb{T}_{h}$, then the action of $M_{h}$ in $T$ is given by

$$
\left.\left(M_{h} p_{h}\right)\right|_{T}=\frac{1}{|T|} \int_{T} p_{h}(x) d x, \quad T \in \mathbb{T}_{h} .
$$

A final observation concerns the unconstrained case, which is $U^{a d}=L^{2}(\Omega)$. In this situation we have $\lambda^{*}=0$ a.e. in $\Omega$. From (25)-(26) we see that the error estimator remains unaffected.

Our investigations concentrate now on the term

$$
\frac{1}{2}\left[\left(b-u_{h}^{*}, \lambda^{*}\right)_{0, \Omega}+\left(b_{h}-u^{*}, \lambda_{h}^{*}\right)_{0, \Omega}\right]=: \Psi^{*}(\Omega),
$$


which contains $u^{*}$ and $\lambda^{*}$. A simple manipulation yields

$$
\begin{gathered}
\Psi^{*}(\Omega)=\frac{1}{2}\left[\left(\lambda_{h}^{*}-\lambda^{*}, b_{h}-u^{*}\right)_{0, \Omega}+\left(\lambda^{*}-\lambda_{h}^{*}, b-u_{h}^{*}\right)_{0, \Omega}\right. \\
\left.+\left(\lambda^{*}-\lambda_{h}^{*}, b_{h}-b\right)_{0, \Omega}\right]
\end{gathered}
$$

From first order optimality we recall

$$
\begin{aligned}
& u^{*} \leq b, \lambda^{*} \geq 0,\left(u^{*}-b, \lambda^{*}\right)_{0, \Omega}=0, \alpha u^{*}-p^{*}+\lambda^{*}=0, \\
& u_{h}^{*} \leq b, \lambda_{h}^{*} \geq 0,\left(u_{h}^{*}-b, \lambda_{h}^{*}\right)_{0, \Omega}=0, \alpha u_{h}^{*}-M_{h} p_{h}^{*}+\lambda_{h}^{*}=0 .
\end{aligned}
$$

Obviously, we have

$$
\begin{aligned}
\Psi^{*}\left(\mathcal{I}^{*} \cap \mathcal{I}_{h}^{*}\right) & =0, \\
\Psi^{*}\left(\mathcal{A}^{*} \cap \mathcal{A}_{h}^{*}\right) & =\frac{1}{2}\left(\lambda^{*}-\lambda_{h}^{*}, b_{h}-b\right)_{0, \mathcal{A}^{*} \cap \mathcal{A}_{h}^{*},}
\end{aligned}
$$

where $\Psi^{*}(\mathcal{S})=\frac{1}{2}\left[\left(b-u_{h}^{*}, \lambda^{*}\right)_{0, \mathcal{S}}+\left(b_{h}-u^{*}, \lambda_{h}^{*}\right)_{0, \mathcal{S}}\right]$. Note that if $b_{h}=b$ a.e. in $\Omega$, then $\Psi^{*}\left(\mathcal{A}^{*} \cap \mathcal{A}_{h}^{*}\right)=0$. From the structure of $\Psi^{*}\left(\mathcal{A}^{*} \cap \mathcal{A}_{h}^{*}\right)$ we can see that it represents a dual weighted data oscillation on $\mathcal{A}^{*} \cap \mathcal{A}_{h}^{*}$. Subsequently we use

$$
\operatorname{osc}_{h}^{\mathcal{S}}\left(b ; \lambda^{*}-\lambda_{h}^{*}\right):=\left(b_{h}-b, \lambda_{h}^{*}-\lambda^{*}\right)_{0, \mathcal{S}} .
$$

Note that $\operatorname{osc}_{h}^{\mathcal{I}^{*} \cap \mathcal{I}_{h}^{*}}\left(b ; \lambda^{*}-\lambda_{h}^{*}\right)=0$.

Utilizing (30)-(33), for $\mathcal{C}_{1}^{*}=\mathcal{A}^{*} \cap \mathcal{I}_{h}^{*}$ and $\mathcal{C}_{2}^{*}=\mathcal{I}^{*} \cap \mathcal{A}_{h}^{*}$ we obtain

$$
\begin{aligned}
\Psi^{*}\left(\mathcal{C}_{1}^{*}\right) & =-\frac{\alpha}{2}\left\|u_{h}-u^{*}\right\|_{0, \mathcal{C}_{1}^{*}}^{2}+\frac{1}{2}\left(p^{*}-M_{h} p_{h}^{*}, u^{*}-u_{h}^{*}\right)_{0, \mathcal{C}_{1}^{*}} \\
\Psi^{*}\left(\mathcal{C}_{2}^{*}\right) & =\frac{1}{2}\left(b_{h}-\alpha^{-1} p^{*}, \lambda_{h}^{*}\right)_{0, \mathcal{C}_{2}^{*}} .
\end{aligned}
$$

On the respective sets we get the following estimates.

(i) In $\mathcal{C}_{1}^{*}$ we have $u_{\mid \mathcal{C}_{1}^{*}}^{*}=b_{\mid \mathcal{C}_{1}^{*}}$. Thus,

$$
\begin{gathered}
\left|\Psi^{*}\left(\mathcal{C}_{1}^{*}\right)\right| \leq \frac{1}{2}\left(\left\|M_{h} p_{h}^{*}-p_{h}^{*}\right\|_{0, \mathcal{C}_{1}^{*}}+\left\|p_{h}^{*}-p^{*}\right\|_{0, \mathcal{C}_{1}^{*}}+\alpha\left\|u_{h}^{*}-b\right\|_{0, \mathcal{C}_{1}^{*}}\right) \cdot \\
\cdot\left\|u_{h}^{*}-b\right\|_{0, \mathcal{C}_{1}^{*}}
\end{gathered}
$$

Given $\mathcal{C}_{1}^{*}$ and the discrete control $u_{h}^{*}$ and adjoint state $p_{h}^{*}$, the first and third terms in parenthesis above are computable a posteriori. We therefore study $\left\|p_{h}^{*}-p^{*}\right\|_{0, \mathcal{C}_{1}^{*}}$ next. Since $p_{h}^{*}, p^{*} \in H_{0}^{1}(\Omega)$ and, for $n \geq 2, H_{0}^{1}(\Omega) \subset L^{s}(\Omega)$ for some $s \in(2,+\infty)$, from Hölder's inequality we obtain

$$
\left\|p_{h}^{*}-p^{*}\right\|_{0, \mathcal{C}_{1}^{*}} \leq \operatorname{meas}\left(\mathcal{C}_{1}^{*}\right)^{r(s)}\left|p^{*}-p_{h}^{*}\right|_{1, \mathcal{C}_{1}^{*}} \leq C_{4} \operatorname{meas}\left(\mathcal{C}_{1}^{*}\right)^{r(s)} \eta_{4},
$$

with $r(s):=\frac{1}{2}-\frac{1}{s}>0$. Hence, we get

$$
\left\|p_{h}^{*}-p^{*}\right\|_{0, \mathcal{C}_{1}^{*}} \leq \min \left(C_{0}^{p} \eta_{0, p}, C_{4} \operatorname{meas}\left(\mathcal{C}_{1}^{*}\right)^{r(s)} \eta_{4}\right)=: C^{p}\left(\mathcal{C}_{1}^{*}\right),
$$


where $\eta_{0, p}$ denotes the a posteriori estimator for $\left\|p^{*}-p_{h}^{*}\right\|_{0, \Omega}$ (see appendix A for its derivation) and $C_{0}^{p}>0$ is a constant. This yields

$$
\begin{gathered}
\left|\Psi^{*}\left(\mathcal{C}_{1}^{*}\right)\right| \leq \frac{1}{2}\left(\left\|M_{h} p_{h}^{*}-p_{h}^{*}\right\|_{0, \mathcal{C}_{1}^{*}}+C^{p}\left(\mathcal{C}_{1}^{*}\right)+\alpha\left\|u_{h}^{*}-b\right\|_{0, \mathcal{C}_{1}^{*}}\right) \cdot \\
\cdot\left\|u_{h}^{*}-b\right\|_{0, \mathcal{C}_{1}^{*}}=: \mu_{1}\left(\mathcal{C}_{1}^{*}\right) .
\end{gathered}
$$

(ii) In $\mathcal{C}_{2}^{*}$ we use the identities $\lambda_{h}^{*}=M_{h} p_{h}^{*}-\alpha u_{h}^{*}$ and $p^{*}=\alpha u^{*}$. From this and assuming $b_{h} \in L^{t}(\Omega), 2 \leq t \leq s$, we infer

$$
\begin{aligned}
2\left|\Psi^{*}\left(\mathcal{C}_{2}^{*}\right)\right| & =\left|\left(u_{h}^{*}-u^{*}, \lambda_{h}^{*}\right)_{\mathcal{C}_{2}^{*}}\right| \\
& \leq \operatorname{meas}\left(\mathcal{C}_{2}^{*}\right)^{r(t)}\left\|b_{h}-\alpha^{-1} p^{*}\right\|_{t, \mathcal{C}_{2}^{*}}\left\|\lambda_{h}^{*}\right\|_{0, \mathcal{C}_{2}^{*}} \\
& \leq \operatorname{meas}\left(\mathcal{C}_{2}^{*}\right)^{r(t)}\left(\left\|b_{h}-\alpha^{-1} p_{h}^{*}\right\|_{t, \mathcal{C}_{2}^{*}}+\alpha^{-1}\left|p_{h}^{*}-p^{*}\right|_{1, \Omega}\right)\left\|\lambda_{h}^{*}\right\|_{0, \mathcal{C}_{2}^{*}} \\
& \leq \operatorname{meas}\left(\mathcal{C}_{2}^{*}\right)^{r(t)}\left(\left\|b_{h}-\alpha^{-1} p_{h}^{*}\right\|_{t, \mathcal{C}_{2}^{*}}+\alpha^{-1} C_{4} \eta_{4}\right)\left\|\lambda_{h}^{*}\right\|_{0, \mathcal{C}_{2}^{*}}
\end{aligned}
$$

with $r(t) \geq 0$. Alternatively, we may use (24a) for estimating $\left\|u_{h}^{*}-u^{*}\right\|_{0, \mathcal{M}_{2}^{*}}$. Hence, setting

$$
C^{u}\left(\mathcal{C}_{2}^{*}\right):=\min \left(\operatorname{meas}\left(\mathcal{C}_{2}^{*}\right)^{r(t)}\left(\left\|b_{h}-\alpha^{-1} p_{h}^{*}\right\|_{t, \mathcal{C}_{2}^{*}}+\alpha^{-1} C_{4} \eta_{4}\right), C_{3} \eta_{3}\right)
$$

we obtain

$$
\left|\Psi^{*}\left(\mathcal{C}_{2}^{*}\right)\right| \leq \frac{1}{2} C^{u}\left(\mathcal{C}_{2}^{*}\right)\left\|\lambda_{h}^{*}\right\|_{0, \mathcal{C}_{2}^{*}}:=\mu_{2}\left(\mathcal{C}_{2}^{*}\right)
$$

Since $\lambda_{h}^{*}=0$ in $\mathcal{I}_{h}^{*}$ we obviously have $\mu_{2}\left(\mathcal{I}_{h}^{*}\right)=0$.

In both cases above we assume $\mu_{1}(\emptyset)=0$ and $\mu_{2}(\emptyset)=0$. Summarizing, we obtain

$$
\begin{aligned}
\left|\Psi^{*}(\Omega)\right| & =\left|\Psi^{*}\left(\mathcal{A}^{*} \cap \mathcal{A}_{h}^{*}\right)+\Psi^{*}\left(\mathcal{C}_{1}^{*}\right)+\Psi^{*}\left(\mathcal{C}_{2}^{*}\right)\right| \\
& \leq \frac{1}{2}\left|\operatorname{osc}_{h}^{\mathcal{A}^{*} \cap \mathcal{A}_{h}^{*}}\left(b ; \lambda^{*}-\lambda_{h}^{*}\right)\right|+\mu_{1}\left(\mathcal{C}_{1}^{*}\right)+\mu_{2}\left(\mathcal{C}_{2}^{*}\right) .
\end{aligned}
$$

An alternative (and possibly coarse) estimate of $\Psi^{*}(\Omega)$ uses the error estimate $\eta_{3}$ and $\left\|\lambda_{h}^{*}\right\|_{0, \mathcal{A}_{h}^{*}}$ only:

$$
\left|\Psi^{*}(\Omega)\right|=\left|\left(\lambda_{h}^{*}-\lambda^{*}, u_{h}^{*}-u^{*}\right)\right| \leq C_{3}^{2} \eta_{3}^{2}=: \mu_{3}(\Omega) .
$$

If the original problem is unconstrained with respect to $u$, then $\lambda^{*}=0$. As a consequence, the first order conditions yield $\alpha u^{*}=p^{*}$, i.e., $u^{*}$ inherits the regularity of $p^{*} \in H_{0}^{1}(\Omega)$. Then we may choose the same ansatz when discretizing $p$ and $u$. Thus, we obtain $\eta_{1}=0$, since $M_{h}$ becomes the identity operator, and-up to data oscillations $-\eta_{2}=\eta_{3}$, and further $\left\|M_{h} p_{h}^{*}-p_{h}^{*}\right\|_{0, \mathcal{C}_{1}^{*}}=$ 0 in $\mu_{1}$.

Finally, we express $\mu_{1}$ and $\mu_{2}$ such that we result in cell oriented error estimates. Let us first consider $\mu_{1}\left(\mathcal{C}_{1}^{*}\right)$. We have

$$
\begin{aligned}
\mu_{1}\left(\mathcal{C}_{1}^{*}\right) & =\frac{1}{2}\left(C^{p}\left(\mathcal{C}_{1}^{*}\right)+\left\|M_{h} p_{h}^{*}-p_{h}^{*}\right\|_{0, \mathcal{C}_{1}^{*}}+\alpha\left\|u_{h}^{*}-b\right\|_{0, \mathcal{C}_{1}^{*}}\right)\left\|u_{h}^{*}-b\right\|_{0, \mathcal{C}_{1}^{*}} \\
& =\frac{1}{2}\left(\hat{C}^{p}\left(\mathcal{C}_{1}^{*}\right)+\hat{C}_{5}\left(\mathcal{C}_{1}^{*}\right)\left\|M_{h} p_{h}^{*}-p_{h}^{*}\right\|_{0, \mathcal{C}_{1}^{*}}^{2}+\alpha\left\|u_{h}^{*}-b\right\|_{0, \mathcal{C}_{1}^{*}}^{2}\right) .
\end{aligned}
$$


Above, we use

$$
\hat{C}_{0}^{p}:=\left\{\begin{array}{cl}
C_{0}^{p} \frac{\left\|u_{h}^{*}-b\right\|_{0, \mathcal{C}_{1}^{*}}}{\eta_{0, p}} & \text { if } \operatorname{meas}\left(\mathcal{C}_{1}^{*}\right) \neq 0 \text { and } \eta_{0, p}>0, \\
0 & \text { if } \operatorname{meas}\left(\mathcal{C}_{1}^{*}\right)=0,
\end{array}\right.
$$

as well as

$$
\hat{C}_{4}\left(\mathcal{C}_{1}^{*}\right):=\left\{\begin{array}{cl}
C_{4} \frac{\left\|u_{h}^{*}-b\right\|_{0, \mathcal{C}_{1}^{*}}}{\eta_{4}} & \text { if } \operatorname{meas}\left(\mathcal{C}_{1}^{*}\right) \neq 0 \text { and } \eta_{4}>0, \\
0 & \text { if } \operatorname{meas}\left(\mathcal{C}_{1}^{*}\right)=0,
\end{array}\right.
$$

and further

$$
\hat{C}_{5}\left(\mathcal{C}_{1}^{*}\right):=\left\{\begin{array}{cl}
\frac{\left\|u_{h}^{*}-b\right\|_{0, \mathcal{C}_{1}^{*}}}{\left\|M_{h} p_{h}^{*}-p_{h}^{*}\right\|_{0, \mathcal{C}_{1}^{*}}} & \text { if meas }\left(\mathcal{C}_{1}^{*}\right) \neq 0 \text { and }\left\|M_{h} p_{h}^{*}-p_{h}^{*}\right\|_{0, \mathcal{C}_{1}^{*}}>0, \\
0 & \text { if } \operatorname{meas}\left(\mathcal{C}_{1}^{*}\right)=0 .
\end{array}\right.
$$

We therefore have

$$
\hat{C}^{p}\left(\mathcal{C}_{1}^{*}\right)=\min \left(\hat{C}_{0}^{p} \eta_{0, p}^{2}, \hat{C}_{4}\left(\mathcal{C}_{1}^{*}\right) \operatorname{meas}\left(\mathcal{C}_{1}^{*}\right)^{r(s)} \eta_{4}^{2}\right) .
$$

Finally, we turn to $\mu_{2}\left(\mathcal{C}_{2}^{*}\right)$. We obtain

$$
\mu_{2}\left(\mathcal{C}_{2}^{*}\right)=\frac{1}{2} \hat{C}^{u}\left(\mathcal{C}_{2}^{*}\right)
$$

with

$$
\hat{C}_{i}\left(\mathcal{C}_{2}^{*}\right):= \begin{cases}C_{i} \frac{\left\|\lambda_{h}^{*}\right\|_{0, \mathcal{C}_{2}^{*}}}{\eta_{i}} & \text { if meas }\left(\mathcal{C}_{2}^{*}\right) \neq 0 \text { and } \eta_{i}>0 \\ 0 & \text { if meas }\left(\mathcal{C}_{2}^{*}\right)=0 .\end{cases}
$$

for $i=3,4$, and

$$
\begin{aligned}
\hat{C}^{u}\left(\mathcal{C}_{2}^{*}\right):=\min & \left(\operatorname { m e a s } ( \mathcal { C } _ { 2 } ^ { * } ) ^ { r ( t ) } \left(\left\|b_{h}-\alpha^{-1} p_{h}^{*}\right\|_{t, \mathcal{C}_{2}^{*}}\left\|\lambda_{h}^{*}\right\|_{0, \mathcal{C}_{2}^{*}}\right.\right. \\
& \left.\left.+\alpha^{-1} \hat{C}_{4}\left(\mathcal{C}_{2}^{*}\right) \eta_{4}^{2}\right), \hat{C}_{3}\left(\mathcal{C}_{2}^{*}\right) \eta_{3}^{2}\right) .
\end{aligned}
$$

We summarize our above findings in the following proposition.

Proposition 2.1. Let the assumptions of Theorem 2.1 be satisfied. Then

$$
\left|\Psi^{*}(\Omega)\right| \leq \min \left(\mu_{1}\left(\mathcal{C}_{1}^{*}\right), \mu_{3}(\Omega)\right)+\min \left(\mu_{2}\left(\mathcal{C}_{2}^{*}\right), \mu_{3}(\Omega)\right)=: \hat{\nu} .
$$

In the case, where the solution of $(\mathrm{P})$ satisfies $u^{*}<b$ a.e. on $\Omega$, we expect that $\hat{\nu} \approx 0$. Indeed, for sufficiently small $h$ we have $\lambda_{h}^{*} \approx 0$ (or even $\lambda_{h}^{*}=0$ ). Thus, $\mu_{2}\left(\mathcal{C}_{2}^{*}\right) \approx 0$ (or $\mu_{2}\left(\mathcal{C}_{2}^{*}\right)=0$ ) holds true. Further, $\mu_{1}\left(\mathcal{C}_{1}^{*}\right)=0$ since $\mathcal{A}^{*}=\emptyset$. Then (39) yields $\hat{\nu} \approx 0$ (or $\hat{\nu}=0$ ). If $(\mathrm{P})$ involves no inequality constraints on $u$, which means that we can set $b \equiv+\infty$ on $\Omega$, then we naturally obtain $\hat{\nu}=0$. Hence, we recover the error estimator for unconstrained optimal control problems; compare [2, 7].

For deriving the full error estimate, it remains to consider the first term in parenthesis on the right hand side of (23) in Theorem 2.2. This term 
is independent of the control constraints and corresponds to the usual expression obtain for (unconstrained) optimal control problems; see [2, 7]. A standard argument yields

$$
\begin{gathered}
\left|\left(\nabla y_{h}^{*}, \nabla\left(i_{h}^{p} p^{*}-p^{*}\right)\right)_{0, \Omega}-\left(u_{h}^{*}+f_{h}, i_{h}^{p} p^{*}-p^{*}\right)_{0, \Omega}\right| \\
\leq \sum_{T}\left\|-\Delta y_{h}^{*}-u_{h}^{*}-f_{h}\right\|_{0, T}\left\|p^{*}-i_{h}^{p} p^{*}\right\|_{0, T} \\
\quad+\sum_{F}\left\|\left[\frac{\partial y_{h}^{*}}{\partial n}\right]\right\|_{0, F}\left\|p^{*}-i_{h}^{p} p^{*}\right\|_{0, F}=: \eta_{2}^{p}
\end{gathered}
$$

for the primal equation,

$$
\begin{gathered}
\left|\left(y_{h}^{*}-z_{h}, i_{h}^{y} y^{*}-y^{*}\right)_{0, \Omega}+\left(\nabla\left(i_{h}^{y} y^{*}-y^{*}\right), \nabla p_{h}^{*}\right)_{0, \Omega}\right| \\
\leq \sum_{T}\left\|-\Delta p_{h}^{*}+y_{h}^{*}-z_{h}\right\|_{0, T}\left\|y^{*}-i_{h}^{y} y^{*}\right\|_{0, T} \\
+\sum_{F}\left\|\left[\frac{\partial p_{h}^{*}}{\partial n}\right]\right\|_{0, F}\left\|y^{*}-i_{h}^{y} y^{*}\right\|_{0, F}=: \eta_{2}^{d}
\end{gathered}
$$

for the dual equation, and

$$
\left|\left(M_{h} p_{h}^{*}-p_{h}^{*}, i_{h}^{u} u^{*}-u^{*}\right)_{0, \Omega}\right|=: \eta_{2}^{u} .
$$

The overall residual and complementarity based error estimate is given in the following theorem.

Theorem 2.3. Let the assumptions of Theorem 2.1 be satisfied. Then we have the following error estimate

$$
\begin{aligned}
\mid J\left(y^{*}, u^{*}\right) & -J\left(y_{h}^{*}, u_{h}^{*}\right) \mid \leq \frac{1}{2}\left(\eta_{2}^{p}+\eta_{2}^{d}+\eta_{2}^{u}+\hat{\nu}\right) \\
& +\frac{1}{2}\left[C_{0}^{p} \eta_{0, p}\left\|f-f_{h}\right\|_{0, \Omega}+C_{0}^{y} \eta_{0, y}\left\|z-z_{h}\right\|_{0, \Omega}\right] \\
& +\left|\operatorname{osc}_{h}\left(x_{h}^{*}\right)\right| .
\end{aligned}
$$

with $\eta_{2}^{p}, \eta_{2}^{d}$, $\eta_{2}^{u}$ and $\hat{\nu}$ defined by (40), (41), (42) and (39), respectively. Further, $C_{0}^{y}>0$ is a constant and $\eta_{0, y}$ denotes an error estimate for $\| y_{h}^{*}-$ $y^{*} \|_{0, \Omega}$. For the definition of $\eta_{0, p}$ and $\eta_{0, y}$ see (62) and (63) in appendix $A$.

The numerical evaluation of (43) depends on estimates of $\left\|i_{h}^{y} y^{*}-y^{*}\right\|_{0, T}$, $\left\|i_{h}^{y} y^{*}-y^{*}\right\|_{0, F}$ and analogously for $i_{h}^{p} p^{*}-p^{*}$. When discretizing the state and the adjoint state in $2 \mathrm{D}$ by continuous piecewise linear finite elements, the following averaging technique replacing $\eta_{2}^{p}$ and $\eta_{2}^{d}$ in (40) and (41), respectively, is appropriate:

$$
\begin{gathered}
\eta_{2, h}^{p}:=\frac{1}{3} \sum_{T}\left(h_{T}\left\|-\Delta y_{h}^{*}-u_{h}^{*}-f_{h}\right\|_{0, T} \sum_{F(T)} h_{F}^{1 / 2}\left\|\left[\frac{\partial p_{h}^{*}}{\partial n}\right]\right\|_{0, F}\right) \\
+\sum_{F} h_{F}\left\|\left[\frac{\partial y_{h}^{*}}{\partial n}\right]\right\|_{0, F}\left\|\left[\frac{\partial p_{h}^{*}}{\partial n}\right]\right\|_{0, F}
\end{gathered}
$$


for the primal equation, and

$$
\begin{gathered}
\eta_{2, h}^{d}:=\frac{1}{3} \sum_{T}\left(h_{T}\left\|-\Delta p_{h}^{*}+y_{h}^{*}-z_{h}\right\|_{0, T} \sum_{F(T)} h_{F}^{1 / 2}\left\|\left[\frac{\partial y_{h}^{*}}{\partial n}\right]\right\|_{0, F}\right) \\
+\sum_{F} h_{F}\left\|\left[\frac{\partial p_{h}^{*}}{\partial n}\right]\right\|_{0, F}\left\|\left[\frac{\partial y_{h}^{*}}{\partial n}\right]\right\|_{0, F}
\end{gathered}
$$

for the dual equation, where $F(T)$ denotes the edges pertinent to triangle $T$. Notice that (44) and (45) yield typically sharper estimates than residualbased estimators for our model problem; compare (24) and [5]. Further observe that we can only expect boundedness of $\left\|i_{h}^{u} u^{*}-u^{*}\right\|_{0, \Omega}$, in general. However, typically $\left\|M_{h} p_{h}^{*}-p_{h}^{*}\right\|_{0, \Omega}$ is small, or, when using the same ansatz for discretizing $u^{*}$ as well as $p^{*}$, it is even zero.

For the numerical evaluation of $\hat{\nu}$ observe that $\mathcal{I}_{h}^{*} \backslash \mathcal{A}^{*} \subset \mathcal{I}^{*}$ and hence $\lambda_{h}^{*}=0$ and $\lambda^{*}=0$ on this set. Consequently, we obtain

$$
\Psi^{*}\left(\mathcal{I}_{h}^{*} \backslash \mathcal{A}^{*}\right)=0
$$

Next observe that $\mathcal{I}_{h}^{*}=\mathcal{C}_{1}^{*} \dot{\cup}\left(\mathcal{I}_{h}^{*} \backslash \mathcal{A}^{*}\right)$. Therefore, we have

$$
\Psi^{*}\left(\mathcal{C}_{1}^{*}\right)=\Psi^{*}\left(\mathcal{I}_{h}^{*}\right)-\Psi^{*}\left(\mathcal{I}_{h}^{*} \backslash \mathcal{A}^{*}\right)=\Psi^{*}\left(\mathcal{I}_{h}^{*}\right) .
$$

If $b_{h}=b$, then we obtain $\Psi^{*}\left(\mathcal{A}_{h}^{*} \backslash \mathcal{I}^{*}\right)=0$ and further

$$
\Psi^{*}\left(\mathcal{C}_{2}^{*}\right)=\Psi^{*}\left(\mathcal{A}_{h}^{*}\right)-\Psi^{*}\left(\mathcal{A}_{h}^{*} \backslash \mathcal{I}^{*}\right)=\Psi^{*}\left(\mathcal{A}_{h}^{*}\right) .
$$

The estimates $\mu_{1}\left(\mathcal{C}_{1}^{*}\right)$ and $\mu_{1}\left(\mathcal{C}_{2}^{*}\right)$, however, do not satisfy relations analogous to (46)-(47) even when $b_{h}=b$. Hence, $\hat{\nu}$ is not a posteriori. In order to have a fully a posteriori estimate we replace $\hat{\nu}$ in (43) by

$$
\hat{\nu}^{a}=\min \left(\mu_{1}\left(\mathcal{I}_{h}^{*}\right), \mu_{3}(\Omega)\right)+\min \left(\mu_{2}\left(\mathcal{A}_{h}^{*}\right), \mu_{3}(\Omega)\right) .
$$

An alternative technique based on set estimation is the subject of section 3.3.

\section{EXTENSIONS}

Now we consider possible extensions of the concept derived in the previous section. We focus on three aspects: (i) Modifications for bilateral constraints; (ii) effects due to nonlinear PDEs; and (iii) alternative ways of making $\hat{\nu}$ fully a posteriori.

3.1. Bilateral constraints. We start by considering the bilaterally constrained version of $(\mathrm{P})$ :

$$
\left\{\begin{array}{l}
\operatorname{minimize} J(y, u):=\frac{1}{2}\|y-z\|_{0, \Omega}^{2}+\frac{\alpha}{2}\|u\|_{0, \Omega}^{2} \\
\text { over } \quad(y, u) \in H_{0}^{1}(\Omega) \times L^{2}(\Omega) \\
\text { subject to }-\Delta y=u+f, \\
\quad a \leq u \leq b \quad \text { a.e. in } \Omega,
\end{array}\right.
$$


Then the first order conditions involve a bilateral complementarity system.

$$
\begin{aligned}
& -\Delta y^{*}=u^{*}+f, \\
& -\Delta p^{*}+y^{*}=z, \\
& \alpha u^{*}+\lambda_{b}^{*}-\lambda_{a}^{*}-p^{*}=0, \\
& u^{*} \geq a, \lambda_{a}^{*} \geq 0,\left(u^{*}-a, \lambda_{a}^{*}\right)_{0, \Omega}=0, \\
& u^{*} \leq b, \lambda_{b}^{*} \geq 0,\left(u^{*}-b, \lambda_{b}^{*}\right)_{0, \Omega}=0 .
\end{aligned}
$$

The analogous relations hold true for the discrete system. The same technique as before yields the bilateral version of Theorem 2.1.

Theorem 3.1. Let $\left(x^{*}, \lambda^{*}\right) \in X \times L^{2}(\Omega)$ and $\left(x_{h}^{*}, \lambda_{h}^{*}\right) \in X_{h} \times L_{h}$ denote the solution of (49) and its finite dimensional counterpart. Then

$$
\begin{gathered}
J\left(y^{*}, u^{*}\right)-J_{h}\left(y_{h}^{*}, u_{h}^{*}\right)=-\frac{1}{2} \nabla_{x x} \mathcal{L}\left(x_{h}^{*}-x^{*}, x_{h}^{*}-x^{*}\right)+\left(u_{h}^{*}-b, \lambda_{b}^{*}\right)_{0, \Omega} \\
+\left(a-u_{h}^{*}, \lambda_{a}^{*}\right)_{0, \Omega}+\operatorname{osc}_{h}\left(x_{h}^{*}\right) .
\end{gathered}
$$

Further we have the following representation; compare Theorem 2.2.

Theorem 3.2. Let the assumptions of Theorem 3.1 be satisfied. Then

$$
\begin{aligned}
J\left(y^{*}, u^{*}\right)- & J_{h}\left(y_{h}^{*}, u_{h}^{*}\right)= \\
= & -\frac{1}{2}\left(\left(y_{h}^{*}-z_{h}, i_{h}^{y} y^{*}-y^{*}\right)_{0, \Omega}+\left(\nabla\left(i_{h}^{y} y^{*}-y^{*}\right), \nabla p_{h}^{*}\right)_{0, \Omega}\right. \\
+ & \left.\left(\nabla\left(i_{h}^{p} p^{*}-p^{*}\right), \nabla y_{h}^{*}\right)_{0, \Omega}-\left(u_{h}^{*}+f_{h}, i_{h}^{p} p^{*}-p^{*}\right)_{0, \Omega}\right) \\
+ & \frac{1}{2}\left[\left(b-u_{h}^{*}, \lambda_{b}^{*}\right)_{0, \Omega}+\left(b_{h}-u^{*}, \lambda_{b, h}^{*}\right)_{0, \Omega}\right] \\
+ & \frac{1}{2}\left[\left(u_{h}^{*}-a, \lambda_{a}^{*}\right)_{0, \Omega}+\left(u^{*}-a_{h}, \lambda_{a, h}^{*}\right)_{0, \Omega}\right]+\frac{1}{2}\left(f-f_{h}, p_{h}^{*}-p^{*}\right)_{0, \Omega} \\
& +\frac{1}{2}\left(z-z_{h}, y_{h}^{*}-y^{*}\right)_{0, \Omega}+\operatorname{osc}_{h}\left(x_{h}^{*}\right) .
\end{aligned}
$$

In the definition of $\hat{\nu}$ (see (39)) we use

$$
\mu_{1}\left(\mathcal{C}_{1}^{*}\right)=\hat{C}^{p}\left(\mathcal{C}_{1}^{*}\right)+\hat{C}_{5}\left(\mathcal{C}_{1}^{*}\right)\left\|M_{h} p_{h}^{*}-p_{h}^{*}\right\|_{0, \mathcal{C}_{1}^{*}}^{2}+\alpha\left\|u_{h}^{*}-c\right\|_{0, \mathcal{C}_{1}^{*}}^{2}
$$

where, in the definitions of $\hat{C}^{p}, \hat{C}_{0}^{p}, \hat{C}_{4}\left(\mathcal{C}_{1}^{*}\right)$ and $\hat{C}_{4}\left(\mathcal{C}_{1}^{*}\right)$, the bound $b$ is replaced by

$$
c(x)= \begin{cases}a(x) & \text { if } x \in \mathcal{I}_{h}^{*} \cap \mathcal{A}_{a}^{*}, \\ b(x) & \text { if } x \in \mathcal{I}_{h}^{*} \cap \mathcal{A}_{b}^{*} .\end{cases}
$$

Here we use

$$
\mathcal{A}_{a}^{*}=\left\{x \in \Omega: u^{*}(x)=a(x)\right\}, \mathcal{A}_{b}^{*}=\left\{x \in \Omega: u^{*}(x)=b(x)\right\}, \mathcal{A}^{*}=\mathcal{A}_{a}^{*} \cup \mathcal{A}_{b}^{*} .
$$

An analogous modification is necessary for the estimator $\mu_{2}\left(\mathcal{C}_{2}^{*}\right)$. 
3.2. Semilinear PDEs. Next we assume that the underlying PDE is semilinear:

$$
A(y)=B u+f,
$$

where the operators $A$ and $B$ induce a semilinear form $a(\cdot)(\cdot)$ and a bilinear form $b(\cdot, \cdot)$, respectively. Hence, the weak form of (51) becomes

$$
a(y)(v)=(f, v)_{0, \Omega}+b(u, v) \quad \forall v \in Y .
$$

For our arguments to follow, we assume that $A$ (resp. $a$ ) is sufficiently often differentiable. The corresponding Lagrange function has the structure

$\mathcal{L}\left(x, \lambda_{a}, \lambda_{b}\right)=J(y, u)+a(y)(p)-(f, p)_{0, \Omega}-b(u, p)+\left(a-u, \lambda_{a}\right)_{0, \Omega}+\left(u-b, \lambda_{b}\right)_{0, \Omega}$

The first order necessary optimality conditions are given by

$$
\begin{aligned}
& A\left(y^{*}\right)-B u^{*}=f, \\
& A^{\prime}\left(y^{*}\right)^{\star} p^{*}+J_{y}\left(y^{*}, u^{*}\right)=0, \\
& J_{u}\left(y^{*}, u^{*}\right)+\lambda_{b}^{*}-\lambda_{a}^{*}-B^{\star} p^{*}=0, \\
& u^{*} \geq a, \lambda_{a}^{*} \geq 0,\left(u^{*}-a, \lambda_{a}^{*}\right)_{0, \Omega}=0, \\
& u^{*} \leq b, \lambda_{b}^{*} \geq 0,\left(u^{*}-b, \lambda_{b}^{*}\right)_{0, \Omega}=0 .
\end{aligned}
$$

As the pointwise control constraints are affine, the error estimator for the nonlinear case is similar to the linear case. This parallels the situation in [2] where the unconstrained case was considered. Due to essentially the same proof arguments as in [2, Proposition 6.1], the following result holds true. In what follows we use

$$
\mathcal{L}_{0}(x)=J(y, u)+a(y)(p)-(f, p)_{0, \Omega}-b(u, p)
$$

and $\mathcal{L}_{0, h}(x)$ for its discrete counterpart.

Theorem 3.3. For a Galerkin finite element discretization of the first order necessary optimality conditions (52) the following relation holds true:

$$
\begin{aligned}
J\left(y^{*}, u^{*}\right)-J_{h} & \left(y_{h}^{*}, u_{h}^{*}\right)=\frac{1}{2} \nabla_{x} \mathcal{L}_{0, h}\left(x_{h}^{*}\right)\left(x^{*}-i_{h} x^{*}\right) \\
& +\frac{1}{2}\left[\left(b-u_{h}^{*}, \lambda_{b}^{*}\right)_{0, \Omega}+\left(b_{h}-u^{*}, \lambda_{b, h}^{*}\right)_{0, \Omega}\right] \\
& +\frac{1}{2}\left[\left(u_{h}^{*}-a, \lambda_{a}^{*}\right)_{0, \Omega}+\left(u^{*}-a_{h}, \lambda_{a, h}^{*}\right)_{0, \Omega}\right] \\
& +\frac{1}{2}\left(\left(f-f_{h}, p_{h}^{*}-p^{*}\right)_{0, \Omega}+\left(z-z_{h}, y_{h}^{*}-y^{*}\right)_{0, \Omega}\right)+\operatorname{osc}_{h}\left(x_{h}^{*}\right) \\
& +r\left(x^{*}, x_{h}^{*}\right)
\end{aligned}
$$

where $r\left(x^{*}, x_{h}^{*}\right)$ denotes the remainder term of a Taylor expansion of $\mathcal{L}_{0}$ about $x_{h}^{*}$. It is bounded by

$$
\left|r\left(x^{*}, x_{h}^{*}\right)\right| \leq \sup _{\bar{x} \in\left[x_{h}^{*}, x^{*}\right]}\left|\nabla_{x}^{3} \mathcal{L}_{0}(\bar{x})\left[x^{*}-x_{h}^{*}\right]^{3}\right| .
$$


3.3. Alternative a posteriori estimate for $\hat{\nu}$. At the end of section 2 we derived an a posteriori estimate for $\hat{\nu}$; recall $\hat{\nu}^{a}$ in (48), where we replaced $\mathcal{C}_{1}^{*}$ by $\mathcal{I}_{h}^{*}$ and $\mathcal{C}_{2}^{*}$ by $\mathcal{A}_{h}^{*}$, respectively. This may give rise to an overestimation of the error term pertinent to the complementarity system. In the following we provide an alternative approach based on set estimation.

Assuming, without loss of generality, $b_{h}=b$, we focus on the unilaterally constrained case and start by considering $\hat{\mu}_{1}\left(\mathcal{C}_{1}^{*}\right)$. For this purpose recall that $\mathcal{C}_{1}^{*}=\mathcal{I}_{h}^{*} \cap \mathcal{A}^{*}$. Similarly to $[6$, Section 3.3$]$ we estimate the continuous active set $\mathcal{A}^{*}$ by

$$
\chi_{h}^{\mathcal{A}^{*}}=1-\frac{b-u_{h}^{*}}{\gamma h^{r}+b-u_{h}^{*}},
$$

where $\gamma$ denotes some (possibly small) positive constant, and $r>0$ is fixed. Note that $\chi_{h}^{\mathcal{A}^{*}}=1$ in $\mathcal{A}_{h}^{*}$. Further let $\chi(\mathcal{S})$ denote the characteristic function of a set $\mathcal{S} \subset \Omega$. We briefly argue that our approximation is useful. In fact, assume that $T \subset \mathcal{A}^{*}$. Then

$$
\left\|\chi\left(\mathcal{A}^{*}\right)-\chi_{h}^{\mathcal{A}^{*}}\right\|_{0, T}=\left\|\frac{b-u_{h}^{*}}{\gamma h^{r}+b-u_{h}^{*}}\right\|_{0, T} \leq \min \left\{1, \gamma^{-1} h^{-r}\left\|u^{*}-u_{h}^{*}\right\|_{0, T}\right\}
$$

which tends to zero whenever $\left\|u^{*}-u_{h}^{*}\right\|_{0, T}=\mathcal{O}\left(h^{q}\right)$ with $q>r$. If $T \in \mathcal{I}^{*}$, then we distinguish two cases:

(i) $T \subset\left\{b-u_{h}^{*}>\gamma h^{\epsilon r}\right\}$ for some $0 \leq \epsilon<1$. Then

$$
\left\|\chi\left(\mathcal{A}^{*}\right)-\chi_{h}^{\mathcal{A}^{*}}\right\|_{0, T}=\left\|\frac{\gamma h^{r}}{\gamma h^{r}+b-u_{h}^{*}}\right\|_{0, T} \leq h^{(1-\epsilon) r} \rightarrow 0 \text { as } h \rightarrow 0 .
$$

(ii) Finally, in the case where $T \in\left\{b-u_{h}^{*} \leq \gamma h^{\epsilon r}\right\}$ we use $T \subset \mathcal{I}^{*}$ and $\left\|u^{*}-u_{h}^{*}\right\|_{0, \Omega} \rightarrow 0$ to conclude that the measure of this set tends to zero as $h \rightarrow 0$.

We therefore use the following approximation of $\chi\left(\mathcal{C}_{1}^{*}\right)$ :

$$
\chi\left(\mathcal{C}_{1}^{*}\right) \approx \chi\left(\mathcal{I}_{h}^{*}\right) \chi_{h}^{\mathcal{A}^{*}}=: \chi_{h}^{\mathcal{C}_{1}^{*}} .
$$

In the definition of $\mu_{1}\left(\mathcal{C}_{1}^{*}\right)$ we then use

$$
\left\|\chi_{h}^{\mathcal{C}_{1}^{*}}\left(u_{h}^{*}-b\right)\right\|_{0, \Omega} \quad \text { instead of }\left\|u_{h}^{*}-b\right\|_{0, \mathcal{C}_{1}^{*}}
$$

and analogously for $\left\|M_{h} p_{h}^{*}-p_{h}^{*}\right\|_{0, \mathcal{C}_{1}^{*}}$. Further, the measure of $\mathcal{C}_{1}^{*}$ is approximated by

$$
\operatorname{meas}\left(\mathcal{C}_{1}^{*}\right) \approx \int_{\Omega} \chi_{h}^{\mathcal{C}_{1}^{*}} d x
$$

The definition of $\mu_{2}$ involves the set $\mathcal{C}_{2}^{*}=\mathcal{A}_{h}^{*} \cap \mathcal{I}^{*}$. Here we employ the approximation

$$
\chi_{h}^{\mathcal{C}_{2}^{*}}:=\chi\left(\mathcal{A}_{h}^{*}\right) \chi_{h}^{\mathcal{I}^{*}}
$$

with $\chi_{h}^{\mathcal{I}^{*}}=1-\chi_{h}^{\mathcal{A}^{*}}$. Then we replace $\left\|\lambda_{h}^{*}\right\|_{0, \mathcal{C}_{2}^{*}}$ by $\left\|\chi_{h}^{\mathcal{C}_{2}^{*}} \lambda_{h}^{*}\right\|_{0, \Omega},\left\|b-\alpha p_{h}^{*}\right\|_{t, \mathcal{C}_{2}^{*}}$ by $\left\|\chi_{h}^{\mathcal{C}_{2}^{*}}\left(b-\alpha^{-1} p_{h}^{*}\right)\right\|_{t, \Omega}$, and

$$
\operatorname{meas}\left(\mathcal{C}_{2}^{*}\right) \approx \int_{\Omega} \chi_{h}^{\mathcal{C}_{2}^{*}} d x
$$


The extension of this concept to the bilaterally constrained case is straight forward.

\section{Appendix A. A POSTERIORI ESTIMATES IN $L^{2}$-NORM}

In this section we derive a posteriori error estimates for $\left\|p^{*}-p_{h}^{*}\right\|_{0, \Omega}$ and $\left\|y^{*}-y_{h}^{*}\right\|_{0, \Omega}$. The subsequent proof technique is based on a combination of the approaches in [5] and [8].

In what follows we assume that $\Omega$ is convex, $b=b_{h}$ a.e. in $\Omega$, and we use $a(y, w)=(\nabla y, \nabla w)_{0, \Omega}$. Given $u_{h}^{*} \in L_{h}$, by $y\left(u_{h}^{*}\right), p\left(u_{h}^{*}\right) \in H_{0}^{1}(\Omega)$ we denote the solutions to

$$
\begin{aligned}
& a\left(y\left(u_{h}^{*}\right), v\right)=\left(f+u_{h}^{*}, v\right)_{0, \Omega}, \\
& a\left(p\left(u_{h}^{*}\right), v\right)=\left(z-y\left(u_{h}^{*}\right), v\right)_{0, \Omega}
\end{aligned}
$$

for all $v \in H_{0}^{1}(\Omega)$. The Poincaré-Friedrichs inequality yields

$$
\begin{aligned}
& \left\|p\left(u_{h}^{*}\right)-p^{*}\right\|_{0, \Omega} \leq c(\Omega)\left\|y\left(u_{h}^{*}\right)-y^{*}\right\|_{0, \Omega}, \\
& \left\|y\left(u_{h}^{*}\right)-y^{*}\right\|_{0, \Omega} \leq c(\Omega)\left\|u_{h}^{*}-u^{*}\right\|_{0, \Omega},
\end{aligned}
$$

where we assume that $y^{*} \in H_{0}^{1}(\Omega)$ satisfies $a\left(y^{*}, v\right)=\left(f+u^{*}, v\right)_{0, \Omega}$ for all $v \in H_{0}^{1}(\Omega)$ and $c(\Omega)$ is a constant depending on the domain $\Omega$ only. Hence, for $p^{*} \in H_{0}^{1}(\Omega)$ satisfying $a\left(p^{*}, v\right)=\left(z-y^{*}, v\right)_{0, \Omega}$ for all $v \in H_{0}^{1}(\Omega)$ we get

$$
\left\|p^{*}-p_{h}^{*}\right\|_{0, \Omega} \leq\left\|p\left(u_{h}^{*}\right)-p_{h}^{*}\right\|_{0, \Omega}+c(\Omega)^{2}\left\|u_{h}^{*}-u^{*}\right\|_{0, \Omega}
$$

Next let us assume that $u^{*}$ respectively $u_{h}^{*}$ satisfy the system

$$
\alpha u^{*}-p^{*}+\lambda^{*}=0 \quad \text { and } \quad \alpha u_{h}^{*}-M_{h} p_{h}^{*}+\lambda_{h}^{*}=0 .
$$

Then we obtain

$$
\begin{aligned}
& \alpha\left\|u^{*}-u_{h}^{*}\right\|_{0, \Omega}^{2} \leq\left(\lambda_{h}^{*}-\lambda^{*}, u^{*}-u_{h}^{*}\right)_{0, \Omega}+\left(p^{*}-p_{h}^{*}, u^{*}-u_{h}^{*}\right)_{0, \Omega} \\
& +\frac{\alpha}{4}\left\|u^{*}-u_{h}^{*}\right\|_{0, \Omega}^{2}+\frac{1}{\alpha}\left\|p_{h}^{*}-M_{h} p_{h}^{*}\right\|_{0, \Omega}^{2} \\
& \leq\left(p^{*}-p_{h}^{*}, u^{*}-u_{h}^{*}\right)_{0, \Omega}+\frac{\alpha}{4}\left\|u^{*}-u_{h}^{*}\right\|_{0, \Omega}^{2} \\
& +\frac{1}{\alpha}\left\|p_{h}^{*}-M_{h} p_{h}^{*}\right\|_{0, \Omega}^{2}
\end{aligned}
$$

since $\left(\lambda_{h}^{*}-\lambda^{*}, u^{*}-u_{h}^{*}\right)_{0, \Omega} \leq 0$. One also has

$$
\left(p^{*}-p\left(u_{h}^{*}\right), u^{*}-u_{h}^{*}\right)_{0, \Omega} \leq 0 .
$$

Hence, we have

$$
\begin{aligned}
\left(p^{*}-p_{h}^{*}, u^{*}-u_{h}^{*}\right)_{0, \Omega} & \leq\left(p\left(u_{h}^{*}\right)-p_{h}^{*}, u^{*}-u_{h}^{*}\right)_{0, \Omega} \\
& \leq \frac{\alpha}{4}\left\|u^{*}-u_{h}^{*}\right\|_{0, \Omega}^{2}+\frac{1}{\alpha}\left\|p_{h}^{*}-p\left(u_{h}^{*}\right)\right\|_{0, \Omega}^{2} .
\end{aligned}
$$

This allows us to continue (56):

$$
\left\|u^{*}-u_{h}^{*}\right\|_{0, \Omega}^{2} \leq \frac{2}{\alpha^{2}}\left\|p_{h}^{*}-p\left(u_{h}^{*}\right)\right\|_{0, \Omega}^{2}+\frac{2}{\alpha^{2}}\left\|p_{h}^{*}-M_{h} p_{h}^{*}\right\|_{0, \Omega}^{2}
$$


Combining the above estimates we result in

$$
\begin{aligned}
\left\|p^{*}-p_{h}^{*}\right\|_{0, \Omega} \leq( & \left.+\frac{\sqrt{2}}{\alpha} c(\Omega)^{2}\right)\left\|p_{h}^{*}-p\left(u_{h}^{*}\right)\right\|_{0, \Omega} \\
& +\frac{\sqrt{2}}{\alpha} c(\Omega)^{2}\left\|p_{h}^{*}-M_{h} p_{h}^{*}\right\|_{0, \Omega}, \\
\left\|y^{*}-y_{h}^{*}\right\|_{0, \Omega} \leq \| & y\left(u_{h}^{*}\right)-y_{h}^{*} \|_{0, \Omega}+\frac{\sqrt{2}}{\alpha} c(\Omega)\left(\left\|p_{h}^{*}-p\left(u_{h}^{*}\right)\right\|_{0, \Omega}\right. \\
& \left.+\left\|p_{h}^{*}-M_{h} p_{h}^{*}\right\|_{0, \Omega}\right) .
\end{aligned}
$$

Utilizing standard $L^{2}$-estimates (see, e.g., [8, Proposition 3.8]) we infer

$$
\begin{gathered}
\left\|y\left(u_{h}^{*}\right)-y_{h}^{*}\right\|_{0, \Omega}^{2} \leq C\left(\sum_{T} h_{T}^{2} \eta_{y, T}^{2}+\sum_{F} h_{F}^{2} \eta_{y, F}^{2}\right)=: C \tilde{\eta}_{0, y}^{2}, \\
\left\|p\left(u_{h}^{*}\right)-p_{h}^{*}\right\|_{0, \Omega}^{2} \leq C\left(\sum_{T} h_{T}^{2} \tilde{\eta}_{p, T}^{2}+\sum_{F} h_{F}^{2} \eta_{p, F}^{2}\right)=: C \tilde{\eta}_{0, p}^{2},
\end{gathered}
$$

where the element and edge residuals are given by

$$
\begin{aligned}
\eta_{y, T} & :=h_{T}\left\|f+u_{h}^{*}\right\|_{0, T}, \\
\eta_{y, F} & :=h_{F}^{1 / 2}\left\|n_{F} \cdot\left[\nabla y_{h}^{*}\right]\right\|_{0, F}, \\
\tilde{\eta}_{p, T} & :=h_{T}\left\|z-y\left(u_{h}^{*}\right)\right\|_{0, T}, \\
\eta_{p, F} & :=h_{F}^{1 / 2}\left\|n_{F} \cdot\left[\nabla p_{h}^{*}\right]\right\|_{0, F}
\end{aligned}
$$

with $n_{F}$ denoting the exterior unit normal of $T$. The triangle inequality yields

$$
\sum_{T} h_{T}^{4}\left\|z-y\left(u_{h}^{*}\right)\right\|_{0, T}^{2} \leq C h^{2} \tilde{\eta}_{0, y}^{2}+2 \sum_{T} h_{T}^{2} \eta_{p, T}^{2}
$$

with the element residual

$$
\eta_{p, T}:=h_{T}^{2}\left\|z-y_{h}^{*}\right\|_{0, T}
$$

Finally we derive the estimate

$$
\begin{aligned}
\| p^{*}- & p_{h}^{*} \|_{0, \Omega} \leq C\left(h^{2} \tilde{\eta}_{0, y}^{2}+\sum_{T} h_{T}^{2} \eta_{p, T}^{2}+\sum_{F} h_{F}^{2} \eta_{p, F}^{2}\right)^{1 / 2} \\
& +\frac{\sqrt{2}}{\alpha} c(\Omega)^{2}\left\|p_{h}^{*}-M_{h} p_{h}^{*}\right\|_{0, \Omega}+\operatorname{osc}_{0, h}(z)+\operatorname{osc}_{0, h}(f) \\
= & : C_{0}^{p} \eta_{0, p}+\operatorname{osc}_{0, h}(z)+\operatorname{osc}_{0, h}(f),
\end{aligned}
$$

where the data oscillations are given by

$$
\begin{aligned}
\operatorname{osc}_{0, h}(z) & =\left(\sum_{T} h_{T}^{2} \operatorname{osc}_{T}(z)^{2}\right)^{1 / 2}, \\
\operatorname{osc}_{T}(z) & =h_{T}\left\|z-z_{h}\right\|_{0, T}
\end{aligned}
$$


and analogously for $\operatorname{osc}_{0, h}(f)$.

The error in the state is estimated a posteriori by

$$
\begin{gathered}
\left\|y^{*}-y_{h}^{*}\right\|_{0, \Omega} \leq \quad C \tilde{\eta}_{0, y}+\frac{\sqrt{2}}{\alpha} c(\Omega)\left(\tilde{\eta}_{0, p}+\left\|p_{h}^{*}-M_{h} p_{h}^{*}\right\|_{0, \Omega}\right) \\
+\operatorname{osc}_{0, h}(f)+\operatorname{osc}_{0, h}(z) \\
=: \quad C_{0}^{y} \eta_{0, y}+\operatorname{osc}_{0, h}(f)+\operatorname{osc}_{0, h}(z) . \\
\operatorname{REFERENCES}
\end{gathered}
$$

\section{REFERENCES}

[1] W. Bangerth, and R. Rannacher. Adaptive Finite Element Methods for Differential Equations. Birkhäuser Publisher, Zürich, 2003.

[2] R. Becker, H. Kapp, and R. Rannacher. Adaptive finite element methods for optimal control of partial differential equations: basic concept. SIAM J. Control Optim., 39 (2000), pp. 113-132.

[3] R. Becker and R. Rannacher. An optimal control approach to error estimation and mesh adaptation in finite element methods. Acta Numerica 2000, Cambridge University Press, 2001, pp. 1-101.

[4] K. Erikson, D. Estep, P. Hansbo, And C. Johnson. Introduction to adaptive methods for differential equations. Acta Numerica 1995, Cambridge University Press, 1995, pp. 105-158.

[5] M. Hintermüller, R.H.W. Hoppe, Y. Iliash, and M. Kieweg. An a posteriori error analysis of adaptive finite element methods for distributed elliptic control problems with control constraints. ESAIM: Control, Optimisation and Calculus of Variations (COCV), forthcoming.

[6] R. Li, W. LiU, H. Ma, and T. Tang. Adaptive finite element approximation for distributed elliptic optimal control problems. SIAM J. Control Optim., 41 (2002), pp. 1321-1349.

[7] W. Liu And N. Yan. A posteriori error estimates for distributed convex optimal control problems. Advances in Computational Mathematics, 15 (2001), pp. 285-309.

[8] R. Verfürth. A Review of A Posteriori Error Estimation and Adaptive MeshRefinement Techniques. Wiley-Teubner, Chichester, England, 1996.

Department of Mathematics and Scientific Computing, University of Graz, Heinrichstrasse 36, A-8010 Graz, Austria.

Institute of Mathematics, University of Augsburg, D-86159 Augsburg, Germany, and Department of Mathematics, University of Houston, Houston, TX 77204-3008, U.S.A. 